\title{
Invasive Maxillary Sinus Aspergillosis in a Child with Acute Lymphoblastic Leukemia: A Case Report
}

\author{
Disha A Padalkar ${ }^{1}$, Sanjay Mankar²
}

\begin{abstract}
Background: Fungal paranasal sinusitis most commonly observed infection in immunocompromised hosts or patients with uncontrolled diabetes mellitus. In immunocompromised hosts like patients with leukemia on chemotherapy, it can become life-threatening infection.

Case description: We present a case report of a 6-year-old male child who presented as a case of febrile neutropenia. Diagnosis of invasive paranasal sinusitis by Aspergillus fumigatus was made and treatment with voriconazole commenced. But due to underlying refractory leukemia the child succumbed.

Conclusion: A high index of suspicion of invasive aspergillosis (IA) should be kept in the mind with careful clinical examination should be done in acute lymphoblastic leukemia (ALL) patients with prolonged duration of neutropenia.

Clinical significance: Importance of early diagnosis of fungal sinusitis and early initiation of treatment in leukemic patients.

Keywords: Diagnosis, Invasive paranasal sinus aspergillosis, Leukemia.

Pediatric Infectious Disease (2021): 10.5005/jp-journals-10081-1298
\end{abstract}

\section{INTRODUCTION}

In immunocompromised people, fungus paranasal sinusitis is becoming more common. The most common fungal infections affecting the sinuses are aspergillosis and mucormycosis. These infections can be invasive or non-invasive. Early diagnosis of invasive fungal infections and initiation of appropriate treatment is very important; because invasive fungal sinusitis may produce diverse intracranial complications which can be life-threatening.

\section{Case Description}

A 6-year-old male child, a case of pre-B cell acute lymphoblastic leukemia (ALL) with bone marrow relapse was admitted for his fifth chemotherapy cycle. He developed febrile neutropenia during the third week of his chemotherapy cycle. His complete blood count revealed hemoglobin of $10.2 \mathrm{~g} / \mathrm{dL}$; total leukocyte count (TLC) $900 / \mathrm{mm}^{3}$, platelet count $<10,000 / \mathrm{mm}^{3}$. Blood culture was sent and meropenem and linezolid were started. Blood culture was sterile. As the patient was persistently febrile, suspecting sepsis in ALL-relapse patient with febrile neutropenia (FN), a presumptive diagnosis of hospital-acquired gram-negative infection or fungal infection was made. Liposomal amphotericin B was also started to treat fungal infection. Later in the course, 10 days after onset of fever he developed a minimal dry cough and nasal stuffiness and also generalized edema with abdominal distension followed by lethargy. For neutropenia and thrombocytopenia, injectable granulocyte colony-stimulating factor and frequent platelet transfusion were given, respectively.

In view of persistent fever, infectious disease reference was done. On examination, pallor and generalized edema with facial swelling were present. He was febrile $\left(101^{\circ} \mathrm{F}\right)$; tachycardia (150/minute) and tachypnea (36/minute) were present. He was maintaining $98 \%$ saturation on room air. Tenderness was present over maxillary sinuses. The abdomen was soft, distended with ascites, and hepatosplenomegaly was present. Another systemic examination was normal. Laboratory work-up showed
1,2Department of Pediatrics, Bharati Vidyapeeth Medical College, Pune, Maharashtra, India

Corresponding Author: Sanjay Mankar, Department of Pediatrics, Bharati Vidyapeeth Medical College, Pune, Maharashtra, India, Phone: +91 9422078779, e-mail: mankarsanjay@gmail.com

How to cite this article: Padalkar DA, Mankar S. Invasive Maxillary Sinus Aspergillosis in a Child with Acute Lymphoblastic Leukemia: A Case Report. Pediatr Inf Dis 2021;3(4):160-162.

Source of support: Nil

Conflict of interest: None

hemoglobin: $7.5 \mathrm{~g} / \mathrm{dL}$, TLC: $200 / \mathrm{mm}^{3}$, platelet count: $30,000 / \mathrm{mm}^{3}$; deranged coagulation profile, and normal liver enzymes with hypoalbuminemia. In view of persistent fever spikes with maxillary sinus tenderness without other localized signs, maxillary sinusitis was suspected. Water's view of paranasal sinuses showed bilateral maxillary fullness. Endoscopic nasal biopsy done for definitive microbiological diagnosis, which showed polypoidal masses in bilateral middle meatus and blackish discoloration of the hard palate, was seen. Ten percent potassium hydroxide $(\mathrm{KOH})$ mount and calcofluor white stain of nasal polyp biopsy and palatal biopsy showed septate fungal hyphae with acute-angled branching (Fig. 1). Histopathology of the biopsied material showed angioinvasion and tissue invasion and culture of the same showed growth of Aspergillus fumigatus (Fig. 2). Liposomal amphotericin B changed to injectable voriconazole. High-resolving CT (HRCT) of paranasal sinuses was done, which showed only soft tissue thickening and fluid in bilateral ethmoidal and maxillary sinuses, nasopharynx and nasal cavity, and no bony erosion. High-resolving CT of the thorax was done to find the coexistence of invasive pulmonary aspergillosis (IPA). It revealed a solitary pulmonary nodule in the posterior basal segment of the right lung, which is one of the commonest findings of IPA.

After initiating voriconazole treatment, hemodynamic condition improved, facial edema and nasal stuffiness decreased 


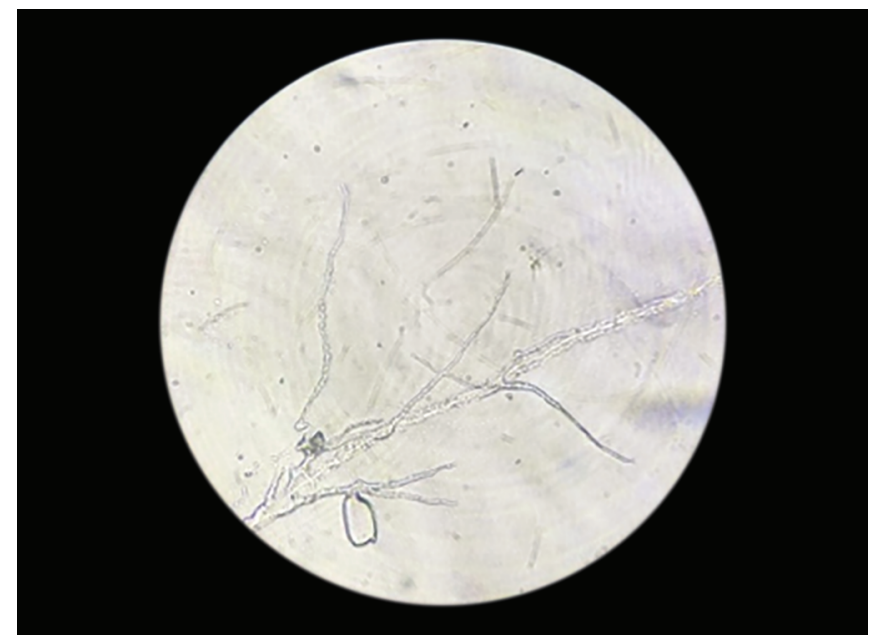

Fig. 1: $\mathrm{KOH}$ mount of palatal tissue showing fungal hyphae

but febrile neutropenia and thrombocytopenia both were persistent. In view of persistent pancytopenia, recurrent epistaxis, bone marrow evaluation was done which showed $80 \%$ of blast cells. Because of the refractory relapsed, ALL patient succumbed after 10 days of diagnosis.

\section{Discussion}

Fungal sinusitis accounts for $6-9 \%$ of all rhinosinusitis. ${ }^{1}$ Fungus widely recovered from invasive fungal sinusitis include Mucor, Aspergillus, Coccidioides, Histoplasma, Candida, Acremonium, Curvularia, Fonsecaea, and Penicillium. ${ }^{2}$ A recent systematic review of invasive fungal sinusitis in children found that the majority of patients (44.6\%) had underlying leukemia and that Aspergillus was the most common organism (47\%) with a mortality rate of $46 \%{ }^{3}$ Aspergillus fumigatus, $A$. flavia, and $A$. niger are the most common Aspergillus species that cause invasive disease in paranasal sinus aspergillosis. Aspergillus flavia is isolated in $>80 \%$ of cases of acute fungal rhinosinusitis in India. ${ }^{4}$

Chronic indolent sinusitis (invasive), acute fulminant sinusitis (invasive), fungal ball (non-invasive), and allergic sinusitis (noninvasive) are the four types of paranasal sinus aspergillosis depending on the immune response of the host to the fungus. ${ }^{5}$ The immunologic background of the infected host determines the form of Aspergillus infection. The invasive disease develops in immunocompromised patients, while allergic disease develops in immunoreactive patients. Because of its variable clinical appearance and difficulty in diagnosing it, fungal invasive sinusitis is likely underdiagnosed, probably due to a reduced inflammatory response in affected patients. ${ }^{6-8}$

Nasal polyps and stagnant secretions, as well as neutropenia, excessive antibiotic usage, uncontrolled diabetes mellitus, human immunodeficiency virus infection, injuries, burns, and radiation therapy, are all predisposing factors that encourage fungal infections throughout the sinuses. ${ }^{9}$ Nasal blockage, nasal obstruction, hyposmia or anosmia, and, if combined with chronic sinusitis, a purulent nasal discharge are the most common signs of paranasal sinus aspergillosis. Postnasal drip, rhinorrhea, facial pain or swelling, headache, and sleep disruption are some of the secondary symptoms..$^{10}$ In our patient, initial presentation was 10 days of febrile neutropenia without any localized symptoms. Symptoms related to sinusitis such as nasal stuffiness and facial

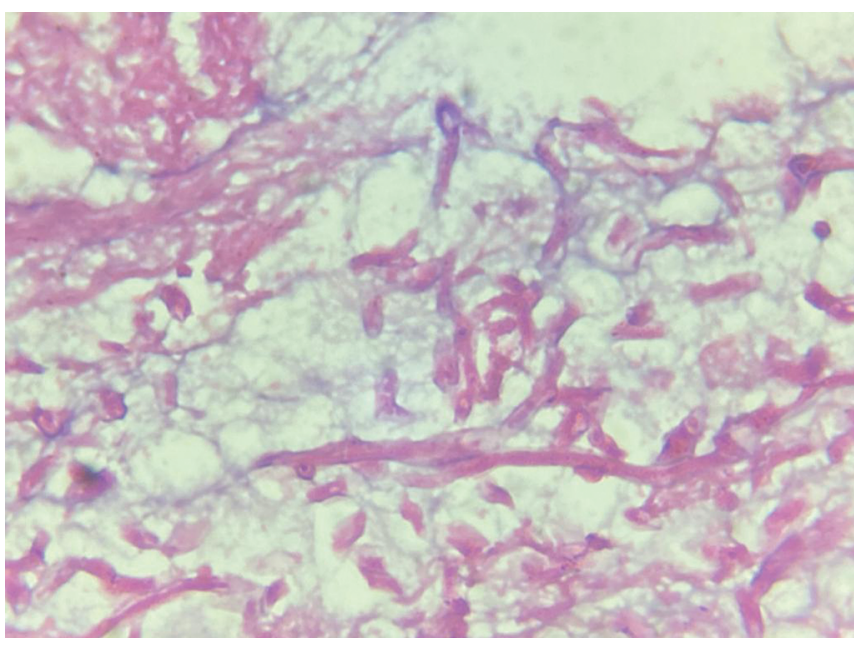

Fig. 2: Histopathology of maxillary sinus mucosa showing tissue invasion and angioinvasion

swelling along with blackish nasal discharge appeared very late in course of the disease. Because of the differences in care and prognosis, it is important to diagnose fungal sinusitis and differentiate between invasive and non-invasive diseases. It is critical to diagnose any fungal sinusitis as soon as possible because delayed treatment can be fatal. This is due to the fungi's proclivity to invade nearby blood vessels, causing thrombosis and, eventually, necrosis of the hard and soft tissue. ${ }^{11}$

Magnetic resonance imaging and computed tomography are used to assist in the diagnosis. The invasive type can display opacity in the sinuses with or without destruction. While bone erosion and extra-sinus extension are classic CT findings indicative of invasive fungal sinusitis, they are typically discovered late in the disease's progression. The most common early sign is severe unilateral nasal cavity mucosal and soft tissue edema. ${ }^{12}$ Endoscopic examination and selective tissue biopsy are used to make a definitive diagnosis. Endoscopy commonly reveals pallor of the mucosa, discoloration or granulation of the mucosa due to ischemia from angioinvasion, and, as the disease progresses, a blackened necrotic focus. Endoscopic evaluation in our patient revealed similar findings.

Ten percent $\mathrm{KOH}$ mount and calcofluor stain of tissue biopsy show hyphae which are typical for each fungus. Aspergillus shows septate hyphae that branch at $45^{\circ}$ angles. ${ }^{1}$ The histology should indicate whether there is mucosal involvement (invasive) or whether the mucosa is intact (non-invasive). To confirm the diagnosis, a fungal culture on Sabouraud's agar is required. In our patient, reports of $10 \% \mathrm{KOH}$ mount, calcofluor stain, and histopathology of tissue from sinus cavity and hard palate were consistent with invasive Aspergillus disease. Culture also revealed the growth of A. fumigatus.

The treatment of invasive aspergillosis is determined by the nature of the disease, host immunity, and the level of tissue invasion. To allow antifungal agents to enter the infected region, surgical debridement of the abnormal sinus tissue is recommended. Surgery can improve fungal disease control and patient survival. The Infectious Disease Society of America (IDSA) released updated guidelines for the treatment of invasive aspergillosis in 2016. In invasive Aspergillus fungal sinusitis, the IDSA suggests surgery as well as systemic voriconazole or a lipid formulation of amphotericin B. We initiated systemic voriconazole therapy 
for the patient. Surgical debridement was not possible in view of underlying severe thrombocytopenia with deranged coagulation profile in relapsed ALL. The prognosis is directly related to the nature of the underlying condition, the magnitude of the disease when treatment starts, and the aggressiveness of the treatment. In our case at the time of diagnosis, the disease was already extended up to the hard palate with possible IPA.

Invasive aspergillosis may have devastating consequences like in our patient. To prevent morbidity and mortality, prophylaxis remains a major goal along with early diagnosis and early initiation of treatment. Infectious Disease Society of America aspergillosis guidelines (2016) recommend "prophylaxis with posaconazole, voriconazole, and/or micafungin during prolonged neutropenia for those who are at high risk for invasive aspergillosis". Patients with hematological conditions with poorly functioning neutrophils (e.g., plastic anemia and variants), acute leukemia with prolonged neutropenia, or a history of invasive aspergillosis (IA) before transplantation are at risk for IA.

\section{ConcLusion}

In ALL patients with chronic neutropenia, a high index of suspicion of IA should be preserved, and a thorough clinical examination should be performed. The secret to effective IA treatment is early detection and clinical intervention, including the selection of an appropriate antifungal agent and surgical debridement.

\section{References}

1. Shmarma $D$, Mahajan $N$, Rao $S$, et al. Invasive maxillary aspergillosis masquerading as malignancy in two cases: utility of cytology as a rapid diagnostic tool. J Cytol 2012;29(3):194-196. DOI: 10.4103/09709371.101171.
2. Marple B, Mabry R. The role of fungus in chronic rhinosinusitis. Curr Opin Otolaryngol Head Neck Surg 2002;10(3):207-212. DOI: 10.1097/00020840-200206000-00011.

3. Smith A, Thimmappa V, Shepherd B, et al. Invasive fungal sinusitis in the pediatric population: systematic review with quantitative synthesis of the literature. Int J Pediat Otorhinolaryngol 2016;90: 231-235. DOI: 10.1016/j.ijporl.2016.09.019.

4. Chatterjee S, Chakrabarti A. Epidemiology and medical mycology of fungal rhinosinusitis. An Int J Otorhinolaryngol Clin 2009;1(1):1-14. DOI: 10.5005/jp-journals-10003-1001.

5. Kamble R, Joshi J, Pendurkar S. Paranasal sinus aspergillosis: a case report and review of literature. Int J Curr Microbiol App Sci 2015;4(9):314-322.

6. Denning D. Invasive aspergillosis. Clin Infect Dis 1998;26(4):781-803. DOI: $10.1086 / 513943$.

7. Ho P, Yuen K. Aspergillosis in bone marrow transplant recipients. Criti Rev Oncol/Hematol 2000;34(1):55-69. DOI: 10.1016/S10408428(00)00047-0.

8. Marr K, Patterson T, Denning D. Aspergillosis. Pathogenesis, clinical manifestations and therapy. Infect Dis Clin N Am 2002;16(4):875-894. DOI: 10.1016/S0891-5520(02)00035-1.

9. Singh N, Siddaraja N, Kumar S, et al. Fine-needle aspiration biopsy as an initial diagnostic modality in a clinically unsuspected case of invasive maxillary fungal sinusitis: a case report. Diagn Cytopathol 2010;38(4):290-293.

10. Panda N, Sharma S, Chakrabartu A, et al. Paranasal sinus mycoses in north India. Mycoses 1998;41(7-8):281-286. DOI: 10.1111/j.14390507.1998.tb00339.x.

11. Taneja T, Saxena S, Aggarwal P, et al. Fungal infections involving maxillary sinus - a difficult diagnostic task. J Clin Experiment Dentis 2011;3(2):e172-e176. DOI: 10.4317/jced.3.e172.

12. DelGaudio J, Swain R, Kingdom T, et al. Computed tomographic findings in patients with invasive fungal sinusitis. Archi Otolaryngol Head Neck Surg 2003;129(2):236. DOI: 10.1001/archotol.129. 2.236 . 